\section{(1) \\ CrossMark}

\title{
Detection of human cytomegalovirus in bronchoalveolar lavage of intensive care unit patients
}

\author{
To the Editor:
}

The seroprevalence of human cytomegalovirus (CMV) is very high worldwide $[1,2]$ and the spectrum of disease caused by it ranges from an asymptomatic state to a mononucleosis-like syndrome to severe diseases such as pneumonia, retinitis or gastrointestinal infection. The most severe disease occurs in congenital infection and in immunosuppressed patients, in whom the virus acts as an opportunistic pathogen. However, the role of CMV in other populations is less clear and is controversial [3]. Some studies in critical patients describe a relationship between CMV and increased mortality rates, longer length of stay and prolonged need for mechanical ventilation [3-5]. The incidence of active CMV infection depends on the diagnostic method used. Several epidemiological studies and systematic reviews have assessed the incidence of CMV infection in mechanically ventilated, critically ill patients, finding values ranging from $0-36 \%$ [5-7]. In this study, we aimed to assess the incidence, clinical characteristics, risk factors and outcomes for intensive care unit (ICU) patients with CMV detection by bronchoalveolar lavage (BAL).

We performed a prospective observational cohort study of consecutive adult patients admitted to two ICUs within $24 \mathrm{~h}$ of admission to the Emergency Department. This study was conducted at the Hospital Clínic of Barcelona (Barcelona, Spain) between January 2013 and November 2015 and the inclusion criteria were: 1) patients must be admitted to the ICU, and 2) appropriate practice of BAL (BAL samples were collected when respiratory infection was suspected and in the presence of bilateral pulmonary infiltrates; range of sampling: $0-8 \mathrm{~h}$ after suspicion). The decision of whether to perform bronchoscopy with BAL was made by the attending physicians. In some cases physicians also ordered PCR in plasma but this test was not performed in all patients. Due to the nature of the study, the researchers had no impact on this decision.

The following parameters were recorded: demographics, comorbidities, immunosuppression status, antibiotic treatment in the previous 30 days before hospital admission, treatment with oral and inhaled corticosteroids, clinical symptoms, laboratory parameters, diagnostic procedures, ventilatory support, length of hospital stay, length of ICU stay and 30-day mortality. Immunosuppression was defined as the presence of solid organ or bone marrow transplantation, HIV infection, cancer under chemotherapy and/ or treatment with corticosteroids (daily doses $>20 \mathrm{mg}$ prednisolone-equivalent for more than 2 weeks in the month prior to admission). The sepsis-related organ failure assessment (SOFA) score was calculated at ICU admission [8]. Samples (BAL and plasma) were collected when respiratory infection was suspected (i.e. observation of new pulmonary infiltrates; range of sampling $0-8 \mathrm{~h}$ after suspicion). The results of BAL were cultured for bacteria, fungi and mycobacteria and $100 \mu \mathrm{L}$ of BAL material were inoculated onto sheep's blood, chocolate, blood charcoal yeast extract (BCYE) and Sabouraud agar. All cultures were incubated at $37^{\circ} \mathrm{C}$ under aerobic conditions and in a carbon dioxide enriched atmosphere (except for Sabouraud agar that was incubated at $25^{\circ} \mathrm{C}$ ). Cultures were evaluated for growth at $24 \mathrm{~h}$ and $48 \mathrm{~h}$ and discarded if results were negative. Bacterial identification and antibiotic susceptibility tests were performed according to European Committee on Antimicrobial Susceptibility Testing (EUCAST) guidelines and breakpoints (version 5.0, 2015; www.eucast.org). Pneumocystis jirovecii was detected by methenamine silver stain while human fibroblast cells were used for the detection of herpes simplex viruses 1 and 2 (HSV-1 and HSV-2) from BAL (monitored for up to 1 week for signs of infection). A BAL was considered positive when a cytopathic effect was observed on conventional cell cultures and then confirmed by bronchoalveolar lavage of intensive care unit patients. Eur Respir J 2018; 51: 1701332 [https://doi.org/ 10.1183/13993003.01332-2017]. 
immunofluorescence detection of the antigen. Molecular detection of CMV in BAL and plasma was performed by real-time quantitative PCR (ELITechGroup S.p.A., Milan, Italy) after extraction of DNA with a DSP Virus/Pathogen Midi Kit on a QIAsymphony automated platform (QIAGEN GmbH, Hilden, Germany). This technique has a detection limit of 20 copies $\cdot \mathrm{mL}^{-1}$ and a quantitative limit of 282 copies $\cdot \mathrm{mL}^{-1}$. Other respiratory viruses were detected by two multiplex reverse transcription nested-PCR assays as previously described [9].

For categorical variables we report the number and percentage of patients while for continuous variables we report the median (interquartile range (IQR)). Categorical variables were compared using the Chi-squared or Fisher exact tests. Continuous variables were compared using the Mann-Whitney test. Logistic regression analyses were performed to identify variables associated with positive detection of CMV and variables that showed a p-value $<0.20$ in the univariate analyses were included in the multivariate model (a backward stepwise procedure). The Hosmer-Lemeshow goodness-of-fit test was performed to assess the overall fit of the multivariate model [10]. The area under the receiver operating characteristic (ROC) curve of the multivariate model to predict positive detection of CMV was calculated. Internal

\begin{tabular}{|c|c|c|c|}
\hline Variable & Negative CMV (n=98) & Positive CMV (n=35) & p-value \\
\hline \multicolumn{4}{|l|}{ Demographic } \\
\hline Age years (median (IQR)) & $62.0(23.0)$ & $61.0(24.0)$ & 0.37 \\
\hline Men & $63(64)$ & $23(66)$ & 0.88 \\
\hline Current smoker & $31(36)$ & $7(25)$ & 0.28 \\
\hline Current alcohol consumer & $8(10)$ & $2(7)$ & $>0.99$ \\
\hline Immunocompromised ${ }^{\#}$ & $47(48)$ & $25(71)$ & 0.017 \\
\hline HIV & $8(8)$ & $3(9)$ & $>0.99$ \\
\hline Transplant & 19 (19) & $9(26)$ & 0.43 \\
\hline Cancer & $16(16)$ & $7(20)$ & 0.62 \\
\hline Systemic corticosteroids ${ }^{\Uparrow}$ & $21(21)$ & $17(49)$ & 0.002 \\
\hline Diagnosis at ICU admission & & & 0.73 \\
\hline Respiratory failure & $59(60)$ & $24(69)$ & \\
\hline Septic shock & $18(18)$ & $5(14)$ & \\
\hline Cardiac failure & $9(9)$ & $2(6)$ & \\
\hline Sepsis & $5(5)$ & $1(3)$ & \\
\hline Other & $7(7)$ & $3(9)$ & \\
\hline Previous antibiotic & $7(7)$ & $3(9)$ & 0.72 \\
\hline Comorbidities $^{+}$ & $76(78)$ & $26(74)$ & 0.70 \\
\hline Chronic respiratory disease & $27(28)$ & $8(23)$ & 0.55 \\
\hline Chronic cardiovascular disease & $23(24)$ & $6(17)$ & 0.44 \\
\hline Diabetes mellitus & $12(12)$ & $6(17)$ & 0.57 \\
\hline Neurological disease & $11(11)$ & 7 (20) & 0.25 \\
\hline Chronic renal disease & $12(12)$ & $7(20)$ & 0.27 \\
\hline Chronic liver disease & $4(4)$ & $2(6)$ & 0.65 \\
\hline Arterial hypertension & 38 (39) & $18(51)$ & 0.19 \\
\hline \multicolumn{4}{|l|}{ Laboratory tests (median (IQR)) } \\
\hline Creatinine $\mathrm{mg} \cdot \mathrm{mL}^{-1}$ & $1.0(1.0)$ & $1.2(1.1)$ & 0.98 \\
\hline C-reactive protein $\mathrm{mg} \cdot \mathrm{dL}^{-1}$ & $12.5(15.9)$ & $11.9(20.0)$ & 0.87 \\
\hline White blood-cell count $10^{9} \cdot \mathrm{L}^{-1}$ & $10.0(9.0)$ & $9.0(12.0)$ & 0.41 \\
\hline SOFA score at ICU admission (median (IQR)) & $5(6)$ & $5(5)$ & 0.91 \\
\hline \multicolumn{4}{|l|}{ Outcomes } \\
\hline Pulmonary complications & 16 (39) & $5(33)$ & 0.70 \\
\hline NIMV & $59(94)$ & $17(94)$ & $>0.99$ \\
\hline IMV & 72 (92) & 26 (93) & $>0.99$ \\
\hline Hospital stay days (median (IQR)) & $35.0(33.0)$ & $46.0(62.0)$ & 0.017 \\
\hline ICU stay days (median (IQR)) & $19.5(21.5)$ & $31.5(69.0)$ & 0.070 \\
\hline 30-day mortality & $38(41)$ & $21(64)$ & 0.024 \\
\hline
\end{tabular}

Data are presented as $\mathrm{n}(\%)$ unless otherwise stated. Values in bold are for when $\mathrm{p}<0.05$. IQR: interquartile range; ICU: intensive care unit; SOFA: sepsis-related organ failure assessment; NIMV: noninvasive mechanical ventilation; IMV: invasive mechanical ventilation. \#: subjects may have more than one immunocompromising condition; ": daily doses $>20 \mathrm{mg}$ prednisolone-equivalent for more than 2 weeks in the month previous to admission; ${ }^{+}$: subjects may have more than one comorbid condition. 
validation was conducted using ordinary nonparametric bootstrapping with 1000 bootstrap samples and bias-corrected, accelerated 95\% confidence intervals (CIs) [11]. The level of significance was set at 0.05 (2-tailed). All analyses were performed using IBM SPSS Statistics 22.0 (Armonk, New York, USA).

During the study period 880 patients were admitted to the two ICUs. BAL was perfomed in 133 patients $(15 \%)$. The three main causes of ICU admission in these 133 patients were: respiratory failure $(n=83$, $62 \%)$, septic shock $(n=23,17 \%)$ and cardiac failure $(n=11,8 \%)$. The main cause for BAL administration was suspected respiratory infection. Detection of CMV was positive in 35 out of 133 BAL samples (26\%) with a median copies. $\mathrm{mL}^{-1}$ value of 7637 (IQR 2604-47249). Testing for CMV was also performed in 19 plasma samples obtained from patients with CMV in BAL testing and results were positive in 13 out of 19 cases $(68 \%)$ with a median copies $\mathrm{mL}^{-1}$ value of 4323 (IQR 433-2272).

In 18 out of $133 \mathrm{BAL}$ procedures (14\%) CMV was the only microorganism detected, while in 17 out of 133 procedures (13\%) CMV and other microorganisms were detected. In 49 out of 133 procedures (37\%) only microorganisms different from CMV were detected and in 49 out of 133 procedures (37\%) no microorganisms were detected. Pseudomonas aeruginosa was the most frequent microorganism isolated (16 out of 133,12\%), followed by Stenotrophomona maltophilia (8 out of 133, 6\%), rhinovirus (7 out of 133, $5 \%)$ and influenza virus A (7 out of 133,5\%). Demographics and clinical characteristics are presented in table 1. Patients with CMV were more likely to have received systemic corticosteroids previously (49\% versus $21 \%, \mathrm{p}=0.002)$, were more frequently immunosuppressed $(71 \%$ versus $48 \%, \mathrm{p}=0.017)$, had longer hospital stays (46 days versus 35 days, $\mathrm{p}=0.017$ ) and had higher 30 -day mortality rates $(64 \%$ versus $41 \%$, $\mathrm{p}=0.024$ ). Multivariate logistic regression analysis revealed that previous use of corticosteroids (OR 3.46, 95\% CI 1.53-7.86) was the only risk factor for positive detection of CMV. The area under the ROC curve was 0.64 (95\% CI $0.52-0.75)$ for the predictive model of positive detection of CMV. The only variable included in the model demonstrated robust results, with a small 95\% CI around the original coefficient.

In testing for CMV in BAL, results were positive in 35 out of 133 ICU patients (26\%) which is a lower incidence than previously published [6]. However, it is remarkable that $29 \%$ of the patients who tested positive for $\mathrm{CMV}$ in BAL were immunocompetent. Immunosuppression was associated with a positive CMV result, which is mainly related to the systemic corticosteroid use. The detection of CMV in BAL was associated with a longer hospital stay and higher mortality, as described previously [3, 12]. The mechanism that could explain this is complex, involving direct CMV pathogenicity [13] or indirect CMV effects [14] such as CMV-mediated immunosuppression [15] and CMV-mediated lung injury. Length of ICU stay was longer in the group of patients with CMV but this was not statistically significant, probably because of the sample size.

All of these studies are observational, which leads us to the question of whether there is a causal relationship between CMV infection and unfavourable outcomes. A CMV-induced mouse infection study showed that anti-CMV treatment improved outcomes in CMV pneumonia; however, few human studies have been carried out to date [16]. One of the limitations of this study and others on this topic is the lack of a cut-off point to determine the number of copies associated with CMV infection. The diagnosis of CMV pneumonitis requires assessment of both microbiological and clinical criteria and without a clear cut-off point it is difficult to distinguish between asymptomatic or latent infection and active infection. Prospective studies comparing different diagnostic techniques and taking into account the treatment and evolution of the patient are thus necessary to standardise the criteria for the diagnosis of CMV pneumonia.

Negative associated outcomes suggest that screening for CMV would be necessary in all ICU patients with suspicion of respiratory infection. However, we stress that detection of CMV by PCR alone may be too sensitive for the diagnosis of CMV pneumonia, as the presence of CMV DNA may be related to pulmonary CMV shedding and there is a possibility of over-diagnosis and over-treatment. Additional prospective trials are necessary to confirm this hypothesis.

Andrea Vergara ${ }^{1,8}$, Catia Cilloniz ${ }^{2,3,8}$, Nestor Luque $^{2}$, Carolina Garcia-Vidal ${ }^{4}$, Javier Tejero ${ }^{5}$, Rafael Perelló ${ }^{6}$, Carmen María Lucena ${ }^{2}$, Antoni Torres ${ }^{2,3}$ and María Angeles Marcos ${ }^{1,7}$

${ }^{1}$ Dept of Clinical Microbiology, Biomedical Diagnostic Center (CDB), Hospital Clínic of Barcelona, University of Barcelona, Institute for Global Health (ISGlobal), Barcelona, Spain. ${ }^{2}$ Dept of Pneumology, Institut Clínic Respiratori (ICR), Hospital Clínic of Barcelona, University of Barcelona, Institut d’Investigacions Biomèdiques August Pi i Sunyer (IDIBAPS), Barcelona, Spain. ${ }^{3}$ SGR 911, Centro de Investigatión Biomédica en Red de Enfermedades Respiratorias (CIBERES), Barcelona, Spain. ${ }^{4}$ Dept of Infectious Diseases, Hospital Clínic of Barcelona, Barcelona, Spain. ${ }^{5}$ University of Barcelona, Barcelona, Spain. ${ }^{6}$ Emergency Dept, Hospital Clínic of Barcelona, Barcelona, Spain. ${ }^{7}$ Barcelona Centre for International Health Research (CRESIB), Institute for Global Health (ISGlobal), Barcelona, Spain. ${ }^{8}$ These authors contributed equally to the study.

Correspondence: María Angeles Marcos, Carrer de Villarroel 170, 08036 Barcelona, Spain.

E-mail: mmarcos@clinic.cat 
Received: July 032017 | Accepted after revision: Nov 302017

Support statement: This work was supported in part by Ciber de Enfermedades Respiratorias (CibeRes CB06/06/0028).

Conflict of interest: None declared.

\section{References}

1 Korndewal MJ, Mollema L, Tcherniaeva I, et al. Cytomegalovirus infection in the Netherlands: seroprevalence, risk factors, and implications. J Clin Virol 2015; 63: 53-58.

2 Bate SL, Dollard SC, Cannon MJ. Cytomegalovirus seroprevalence in the United States: the national health and nutrition examination surveys, 1988-2004. Clin Infect 2010; 50: 1439-1447.

3 Limaye AP, Kirby KA, Rubenfeld GD, et al. Cytomegalovirus reactivation in critically ill immunocompetent patients. JAMA 2008; 300: 413-422.

4 Chiche L, Forel J-M, Roch A, et al. Active cytomegalovirus infection is common in mechanically ventilated medical intensive care unit patients. Crit Care Med 2009; 37: 1850-1857.

5 Frantzeskaki FG, Karampi E-S, Kottaridi C, et al. Cytomegalovirus reactivation in a general, nonimmunosuppressed intensive care unit population: incidence, risk factors, associations with organ dysfunction, and inflammatory biomarkers. J Crit Care 2015; 30: 276-281.

6 Osawa R, Singh N. Cytomegalovirus infection in critically ill patients: a systematic review. Crit Care 2009; 13: R68.

7 Kalil AC, Florescu DF. Prevalence and mortality associated with cytomegalovirus infection in nonimmunosuppressed patients in the intensive care unit. Crit Care Med 2009; 37: 2350-2358.

8 Vincent JL, Moreno R, Takala J, et al. The SOFA (Sepsis-related Organ Failure Assessment) score to describe organ dysfunction/failure. Intensive Care Med 1996; 22: 707-710.

9 Coiras MT, Pérez-Breña P, García ML, et al. Simultaneous detection of influenza A, B, and C viruses, respiratory syncytial virus, and adenoviruses in clinical samples by multiplex reverse transcription nested-PCR assay. $J$ Med Virol 2003; 69: 132-144.

10 Hosmer DW, Lemeshow S. Applied Logistic Regression. New York, Wiley, 1989.

11 Efron B, Tibshirani RJ. Monographs on statistics and applied probability 57: an introduction to the bootstrap. Boca Raton, Chapman \& Hall/CRC, 1994.

12 Lachance $\mathrm{P}$, Chen J, Featherstone R, et al. Impact of cytomegalovirus reactivation on clinical outcomes in immunocompetent critically ill patients: protocol for a systematic review and meta-analysis. Syst Rev 2016; 5: 127.

13 Heininger A, Jahn G, Engel C, et al. Human cytomegalovirus infections in nonimmunosuppressed critically ill patients. Crit Care Med 2001; 29: 541-547.

14 Ljungman P, Griffiths P, Paya C. Definitions of cytomegalovirus infection and disease in transplant recipients. Clin Infect Dis 2002; 34: 1094-1097.

15 Kalil AC, Florescu DF. Is cytomegalovirus reactivation increasing the mortality of patients with severe sepsis? Crit Care 2011; 15: 138.

16 Cook $\mathrm{CH}$, Zhang Y, Sedmak DD, et al. Pulmonary cytomegalovirus reactivation causes pathology in immunocompetent mice. Crit Care Med 2006; 34: 842-849. 